\title{
Isotope-Resolved Electron Energy Loss Spectroscopy in a Monochromated Scanning Transmission Electron Microscope
}

\author{
Jordan A. Hachtel, ${ }^{1+\star}$ Jacob R. Jokisaari, ${ }^{2 \dagger}$ Ondrej L. Krivanek, ${ }^{3}$ Juan Carlos Idrobo, ${ }^{1}$ and \\ Robert F. Klie ${ }^{2}$ \\ ${ }^{1}$ Center for Nanophase Materials Sciences, Oak Ridge National Laboratory, Oak Ridge, TN 37831 \\ ${ }^{2}$ Department of Physics, University of Illinois at Chicago, Chicago, IL 60607 \\ ${ }^{3}$ Nion R\&D, 11511 NE $118^{\text {th }}$ St., Kirkland, WA 98034
}

${ }^{\dagger}$ Authors contributed equally

*hachtelja@ornl.gov

\begin{abstract}
The development and implementation of high-stability monochromators in state-of-the-art aberration-corrected scanning transmission electron microscopes has enabled materials characterization with an energy resolution as good as $3 \mathrm{meV}$. This allows the vibrational modes, which would otherwise be obscured by the energy spread of the electron beam, to be probed with very high precision in molecular materials. Since the vibrational energies depend on the weight of the atomic nuclei, vibrational spectroscopy can distinguish isotopes whose only difference lies in their neutron content. This opens up isotopic analysis and mapping in transmission electron microscopy as two important new research areas. Here, we review the monochromated electron energy loss spectroscopy (EELS) instrumentation, discuss optimal methods for probing beam-sensitive materials without destroying them, and review key nanoscale isotope-resolved results.
\end{abstract}

Keywords: electron energy loss spectroscopy, scanning transmission electron microscopy, vibrational spectroscopy, monochromated aberration correction, liquid cells

\section{Introduction}

Electron monochromators in the scanning transmission electron microscope (STEM) have improved the energy resolution for electron energy loss spectroscopy (EELS) from $300 \mathrm{meV}$ in standard cold field emission guns down to $3 \mathrm{meV}$ [1]. This unprecedented degree of energy resolution allows direct access to a variety of physical phenomena including phonons [2,3], phonon-polaritons [4-6], infrared plasmons [7-9], and molecular vibrations [10-13]. This leads to new experimental capabilities for STEM, such as isotope-resolved electron microscopy. The electron beam itself is insensitive to the chargeless neutrons that define isotopes, and as a result isotopes could only be studied in a STEM using low-efficiency experiments, such as systematic measurements of knock-on damage or Compton scattering $[14,15]$. However, vibrational energies are dependent on the atomic mass of the elements, not just the atomic number. As a result, the energy-loss of the vibrational modes can be directly related to the element's isotopic character.

Furthermore, one can take advantage of different types of scattering mechanisms to access different parts of the vibrational response, as shown in Figure 1. One can collect electrons that are scattered out to high angles to primarily access highly localized impact scattering losses (Figure 2a), which enable phonons to be measured with atomic resolution $[16,17]$ and even the direct measurement of single-atom vibrational modes [18]. Alternatively, the beam can also interact with the dipole moments in the sample and excite optical phonons, leading to delocalized dipole scattering (Figure 2b), enabling efficient excitation even when the electron beam passes tens of nanometers away from the sample in an "aloof" scattering geometry $[10,12,19,20]$. The strength of the aloof interaction is inversely proportional to the excitation energy [21], so at small impact parameters, (tens of $\mathrm{nm}$ ) IR vibrational modes that carry the isotopic information can be excited without subjecting the sample to damaging $\mathrm{UV}+$ radiation. Thus, one can use the aloof mode for long acquisitions that would normally destroy a beam-sensitive material to perform damagefree nanoscale spectroscopy.

Here, we review recent results of isotopically resolved studies on beam-sensitive materials using the monochromated aberration-corrected STEM (MAC-STEM) at Oak Ridge National Laboratory (ORNL). The frequency of these vibrations was probed in the aloof mode with sub-meV precision and was able to identify molecular and isotopic species with nanometer resolution without damaging the sample. The technique opens the door for real-space tracking of isotopically labeled compounds, with vast implications for following nanoscale chemical reactions and metabolic pathways in soft matter and even living organisms.

\section{Beam-Sensitive Materials for Vibrational Spectroscopy}

Water, or liquid $\mathrm{H}_{2} \mathrm{O}$, is arguably the most important chemical compound on earth and is the main component of all living organisms. Deuterated (heavy) water, $\mathrm{D}_{2} \mathrm{O}$, is a widely applied isotopic tracer that has been extensively used in macroscopic vibrational spectroscopy to identify the resultants of chemical reactions and to track functional groups [22]. Water, both $\mathrm{H}_{2} \mathrm{O}$ and $\mathrm{D}_{2} \mathrm{O}$, have been extensively studied by various vibrational spectroscopy techniques [23,24], making it an excellent reference case for budding vibrational EELS.

In order to examine liquid water in the microscope it must be protected both from the vacuum, which can evaporate the liquid, and the electron beam, which can induce radiolysis and create new species including hydrated electrons and hydrogen gas [25]. Recent advances in preparation of liquid cells (LCs) for use in TEM are based on thin layers of graphene [26] and hexagonal boron nitride (hBN) [11] that encapsulate the liquid, 
(a)

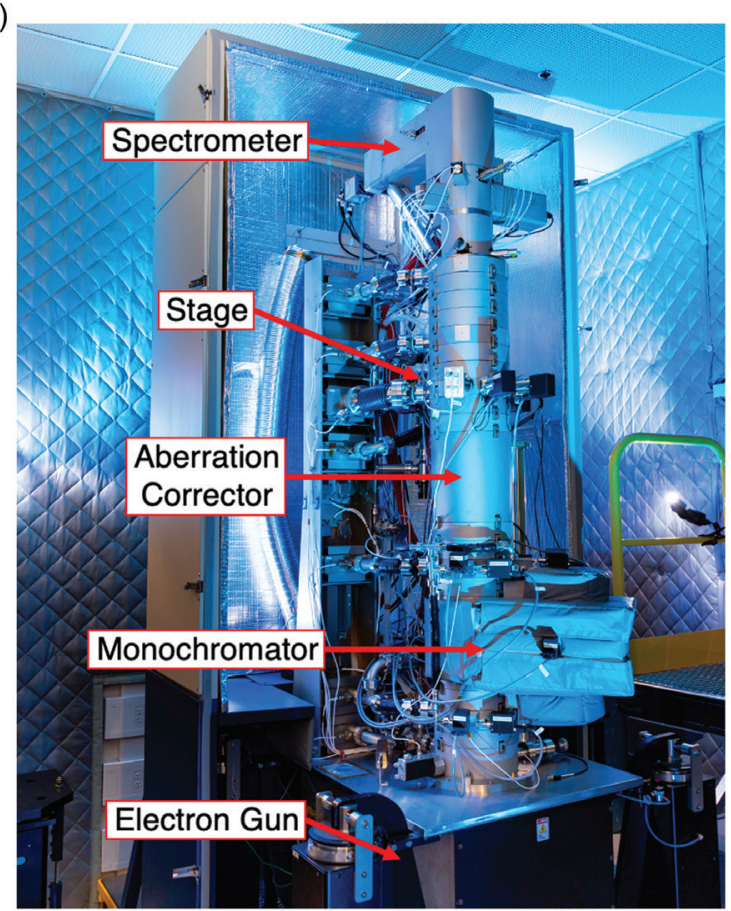

(b)

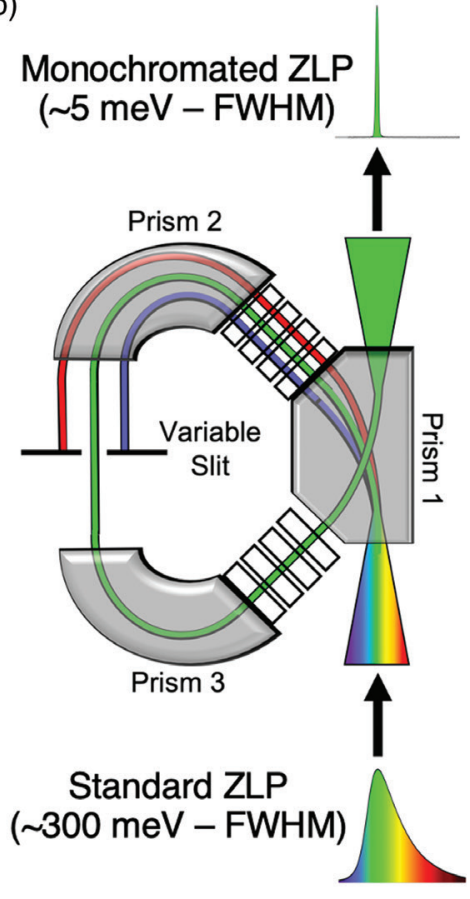

(c)
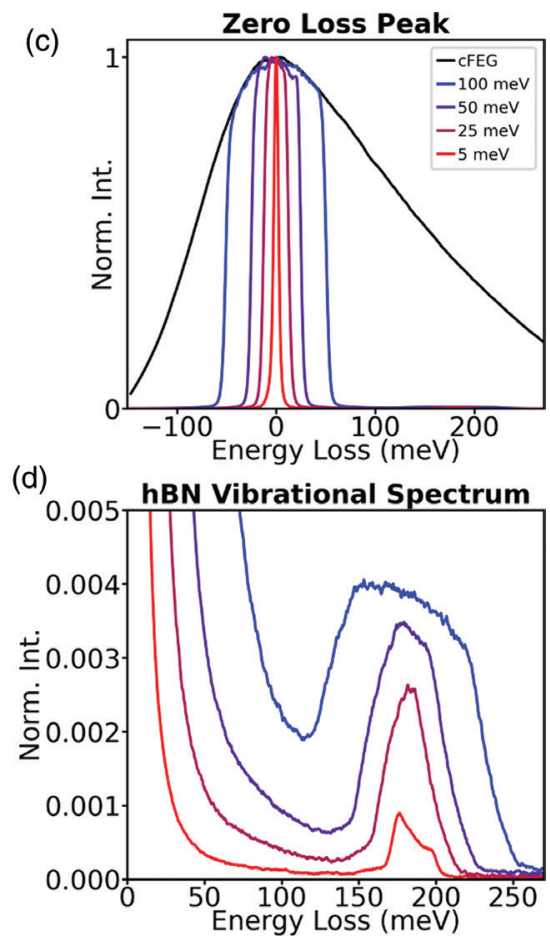

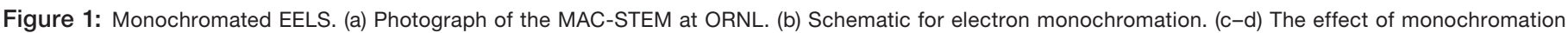

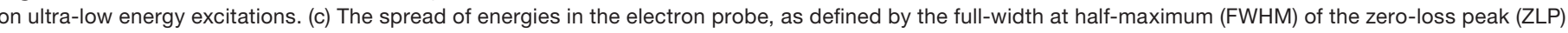

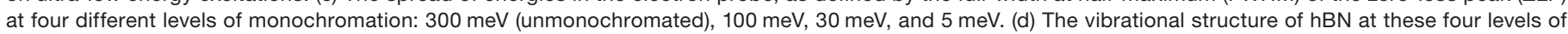
monochromation.

making it vacuum-compatible. The LCs are ideal for spectroscopy as the window thickness and liquid volume are both extremely small compared to traditional SiN liquid-cell TEM holders, enabling a higher fraction of the EELS signal to come from the sample, as opposed to the window. For many imaging applications, the rigidity of graphene is preferable for the LC material, but as a conductor graphene has a continuum of background states in the ultra-low loss EELS region that can obscure the weak vibrational signals. Thus, for vibrational EELS, boron nitride liquid cells (BNLCs) are optimal for isotopically sensitive EELS as they have a clean background in the spectral region of the water and heavy water vibrational modes.

For the BNLCs discussed here, CVD-grown hBN on copper foil was obtained from commercial sources. After etching the copper support, the hBN layer was cleaned several times in pure water and transferred to a copper TEM mesh grid. This was dried, and the water and heavy water samples were dropcast onto the hBN film. Subsequently, a top layer of BN was added to encapsulate the liquid sample by dipping the grid onto another $\mathrm{hBN}$ layer that was floating on $\mathrm{H}_{2} \mathrm{O}$ or $\mathrm{D}_{2} \mathrm{O}$, depending on the sample.

Another example of beam-sensitive materials that are highly relevant in the biological sciences are amino acids, since they are the fundamental building blocks that guide biological processes [27]. In this context, we will review our recent study of crystalline clusters of L-alanine with different isotopic labels. The samples were prepared from commercial powders that were ground to nanoscale clusters with a mortar and pestle and then dispersed on a TEM grid. The clusters ranged from $100 \mathrm{~nm}$ to several microns but were sparse enough for ample vacuum between clusters, allowing singling out of individual clusters for aloof spectroscopy.

\section{Monochromated Electron Energy Loss Spectroscopy in the STEM}

The NION high-energy resolution monochromated EELSSTEM (HERMES) [28] (Figure 1a), which allows for nanoscale vibrational spectroscopy in the electron microscope, was used for the studies summarized here. The major components of

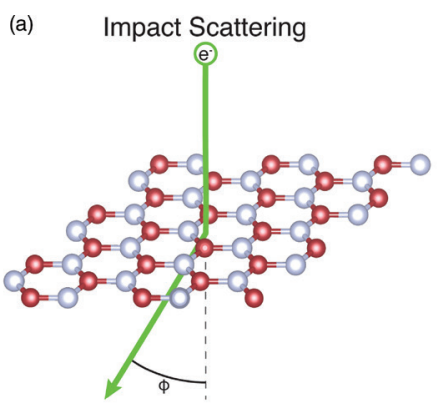

Delocalization: $\leq 0.1 \mathrm{~nm}$

Dominant at Large Scattering Angles Application: Atomic Resolution Spectroscopy (b) Dipole Scattering

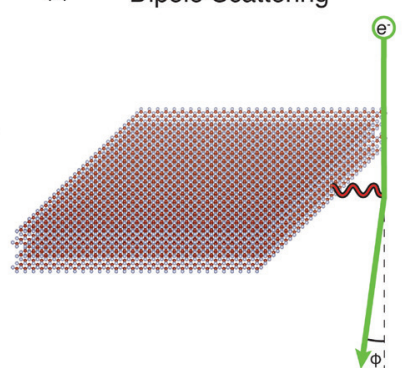

Delocalization: $>10 \mathrm{~nm}$ Dominant Along Optic Axis Application: High-Signal, Low-Damage
Figure 2: Scattering mechanisms for vibrational spectroscopy. Fast electrons can excite vibrational modes through different means. (a) By scattering off of the nucleus of individual electrons, the beam can undergo highly localized impact scattering for atomic-resolution analysis. (b) By interacting with a polar material through a virtual photon, the beam can undergo delocalized dipole scattering for efficient, damage-free analysis. Moreover, impact scattering is dominant at high scattering angles, while dipole scattering is only dominant close to the optic axis, enabling microscopists to switch between the two effects by changing the position of the EELS collection aperture. 
(a)

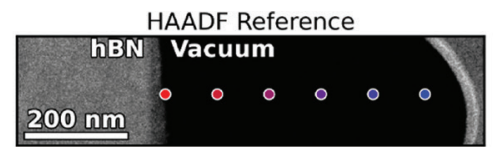

(b)

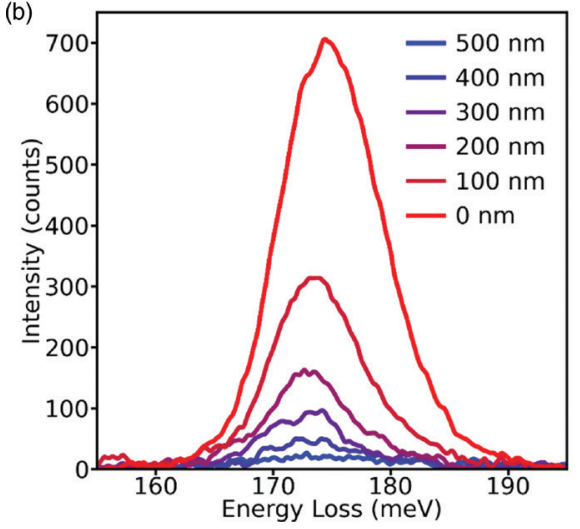

(c)

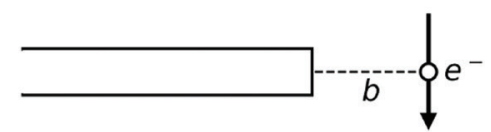

(d)

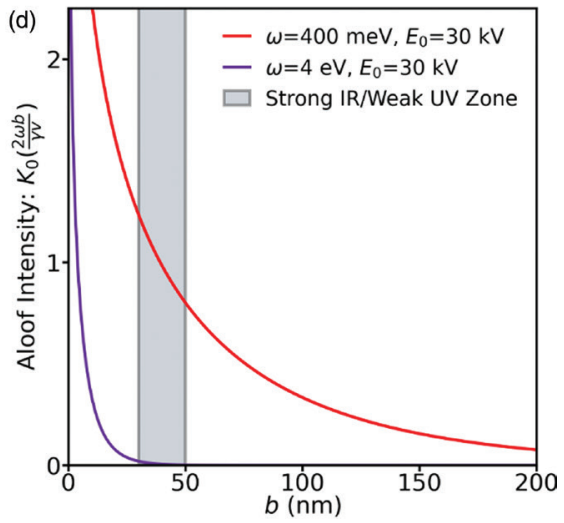

Figure 3: Aloof excitations in monochromated EELS. (a) Dark-field image of a flake of hBN. (b) The aloof spectrum acquired of the BN phonon response from different impact parameters. (c) Schematic of an aloof interaction. (d) The aloof interaction strength as a function of impact parameter and excitation frequency, demonstrating the capacity to probe infrared excitations efficiently without activating damaging UV phenomena.

the sample [2]. This is demonstrated in Figure 3, which shows a dark-field image of an hBN flake (Figure 3a) and several spectra acquired at different distances away from the sample (Figure $3 b$ ). We see that as we get further away from the sample the vibrational intensity drops, but it is still detectable even from $500 \mathrm{~nm}$ away from the sample. This is due to the fact that the electric field of the beam extends far beyond the actual beam trajectory and can still interact with phonons. The strength of the interaction between the beam and a sample (as schematically shown in Figure 3c) can be expressed as the following,

$$
I_{\text {aloof }}(b, \omega, v) \propto K_{0}\left(\frac{2 \omega b}{\gamma v}\right)
$$

where $b$ is the impact parameter of the electron beam (distance from the sample), $\omega$ is the frequency of the excitation, $v$ is the velocity of the electron (in terms of the

the microscope from bottom to top are the electron gun, the monochromator, the aberration-corrector, the stage (location of the sample), and the spectrometer. This configuration allows for us to achieve an electron probe at the sample that is both monochromatic and aberration-free, enabling simultaneously high spatial- and spectral-resolution. While the aberrationcorrector is critical to atomic-resolution studies, the aloof experiments summarized in this article do not require subÅngstrom resolution, so we will focus on the monochromator.

The principle of monochromation is demonstrated schematically in Figure 1b. The HERMES uses a dispersing-undispersing $\alpha$-style monochromator, consisting of two identical halves related by mirror symmetry. In the first half, the electron beam traverses two magnetic prisms, and the electrons are dispersed as a function of energy. A variable slit, placed in the midplane of the monochromator, permits only a narrow band of energies to pass through. The beam is then energy-undispersed in the second half of the monochromator and reinserted in the microscope column as an undispersed beam of greatly reduced energy [29].

Figures 1c and 1d show the low-loss EEL spectrum for hBN at different levels of monochromation with the elastic scattering peak or zero-loss peak (ZLP) in Figure $1 \mathrm{c}$ and the hBN phonon response shown in Figure 1d. The monochromation has two effects: it cuts off the extended low-energy tail of the cold field emission energy distribution (enabling the hBN phonons to be detected clearly, even with a modest energy resolution of $100 \mathrm{meV}$ ) and improves the energy resolution of all features in the spectrum. An energy resolution of $5 \mathrm{meV}$ yields the correct shape of the vibrational peaks and allows many modes tightly bunched together to be individually resolved.

\section{Damage-Free Aloof Spectroscopy}

Aloof excitation of the vibrational signal was first demonstrated in 2014 and has since become a prized technique for analysis of beam-sensitive samples due to the ability to observe vibrational excitations without directly irradiating accelerating voltage of the electron microscope), and $K_{0}$ is a modified Bessel function of the second kind [21].

The $K_{0}$ Bessel function results in an inverse quadraticlike decay as a function of impact parameter (as one might expect for an electromagnetic interaction), which shows why the intensity of the phonon response decays (but does not go away) in Figure 3b. However, we also see that the interaction strength is inversely proportional to the frequency. This is especially critical for organic materials where the higher energy excitations (that is, $>3.5 \mathrm{eV}$ ) bleach molecules and break apart bonds [30], but excitations at the level of molecular vibrations do not damage the material [10]. In Figure 3d the aloof interaction strength as a function of impact parameter for an IR and an UV excitation is plotted, and the UV excitation strength is only significant very close to the sample. Thus, a regime where the beam is a small (but non-zero) distance from the sample $(\sim 30-50 \mathrm{~nm})$ can be defined, exciting the ultra-low IR phenomena effectively without activating the UV excitations that will damage the sample. In this "sweet spot" we can acquire spectra for long periods of time to achieve excellent signal-to-noise without damaging sensitive organic materials, enabling the precise measurement of the vibrational fine structure in molecules.

\section{Isotope-Resolved Spectroscopy in Liquid Water}

The first demonstration of isotope-resolved vibrational EELS was reported for liquid-water BNLCs [11], a schematic of which is presented in Figure $4 \mathrm{a}$. These liquid cells are generally between 25 and $50 \mathrm{~nm}$ thick, and the aloof spectra are recorded and shown in Figure $4 \mathrm{~b}$, enabling the clear detection of the $\mathrm{O}-\mathrm{H}$ stretch mode. We can also use aloof EELS on a BNLC filled with heavy water, the spectra of which is also shown in Figure $4 \mathrm{~b}$. Here, we observe the signals from both the $\mathrm{O}-\mathrm{H}$ stretching mode and the $\mathrm{O}-\mathrm{D}$ stretching modes. The presence of the $\mathrm{O}-\mathrm{H}$ stretching peak in the $\mathrm{D}_{2} \mathrm{O}$ is due to mixing of the specimen, and in part due to the atmospheric water deposited during sample preparation. The spectrum compares well with the Raman data in Figure 4c, 

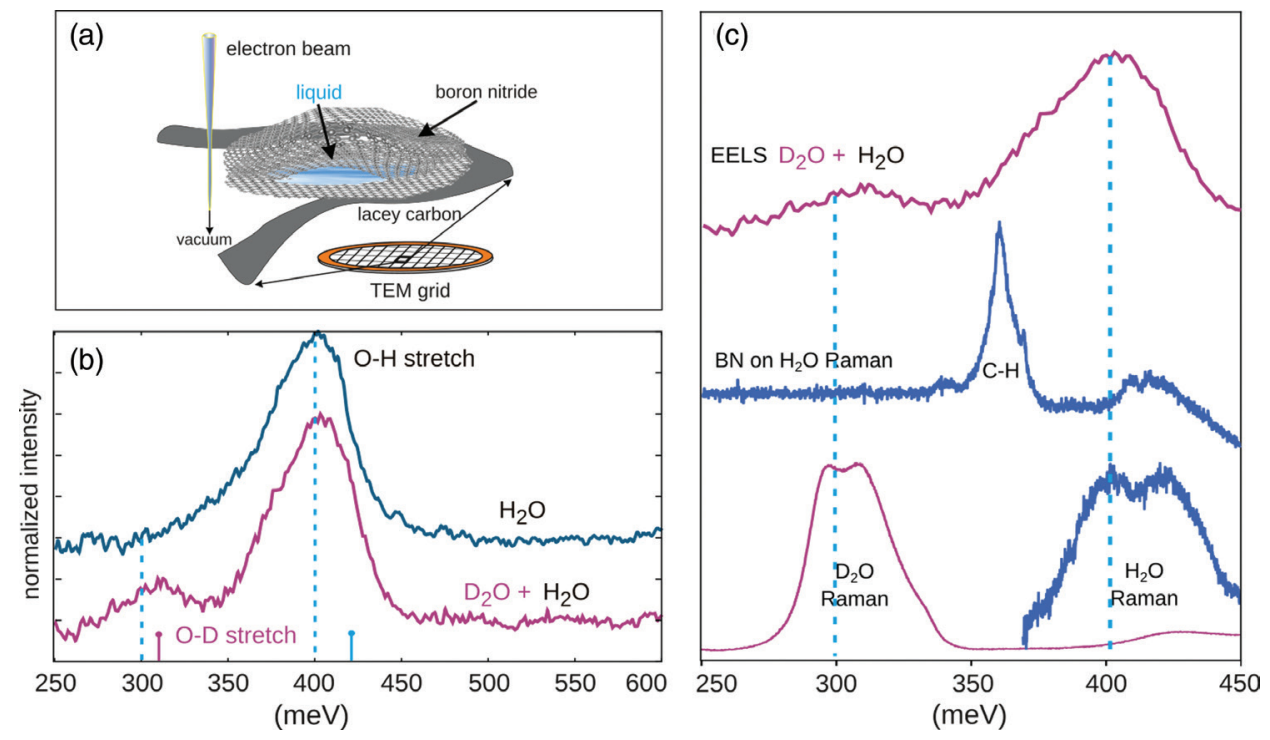

Figure 4: (a) Schematic of a liquid cell. (b) EEL spectra of $\mathrm{H}_{2} \mathrm{O}$ liquid cells and $\mathrm{D}_{2} \mathrm{O}+\mathrm{H}_{2} \mathrm{O}$ liquid cells. (c) Comparison of EEL spectra, Raman of BN floating on water after etching, and Raman of pure $\mathrm{D}_{2} \mathrm{O}$ and $\mathrm{H}_{2} \mathrm{O}$ droplets. Figure adapted from [11].

using BN floating on water, as well as Raman spectra collected from pure $\mathrm{D}_{2} \mathrm{O}$ and $\mathrm{H}_{2} \mathrm{O}$ specimens. The floating $\mathrm{BN}$ does show a $\mathrm{C}-\mathrm{H}$ band, likely resulting from residue from the etching process. However, there was no evidence of this in the EEL spectrum.

There is a clear separation of the O-D and O-H peaks data, enabling the direct detection of $\mathrm{D}_{2} \mathrm{O}$ in the liquid cell. In fact, the difference is significant enough to indicate the potential for isotope-labeling using heavier atoms, with smaller differences in mass between different isotopes, particularly as the technique continues to evolve.

\section{Isotope-Resolved \\ Spectroscopy in \\ Biomolecules}

The change in mass between hydrogen and deuterium (D) is significant and results in a measurable shift of the stretching mode, however, the ultra-high resolution of the microscope affords us the precision to see even more subtle changes. This was observed by isotopically labeling the amino acid L-alanine [12], which has 13 atoms and 4 different elements, meaning isotopiclabeling can take many different forms.

In Figure 5, we show the vibrational EEL spectra, acquired with an energy resolution of $\sim 6 \mathrm{meV}$, from samples with different isotopic labels, and compare them to macroscopic FTIR measurements. We specifically examine the naturally occurring L-alanine (Figure $5 \mathrm{a}),{ }^{13} \mathrm{C}$-labeling in the carboxyl group (Figure $5 b$ ), and deuterium $\left({ }^{2} \mathrm{H}\right)$-labeling in the methyl group (Figure $5 \mathrm{c}$ ). For each sample, we show the chemical formula (i) and the vibrational spectra for EELS and FTIR for the spectral range between 80 and $220 \mathrm{meV}$ (ii-EELS, iii-FTIR) and the spectral 220 and $420 \mathrm{meV}$ (iv-EELS, v-FTIR).

The vibrational response is clearly more complex than water, with dozens of peaks in the low-energy range and a continuum of excitations in the higher-energy range where we observed only the isolated $\mathrm{O}-\mathrm{H}$ and $\mathrm{O}-\mathrm{D}$ stretch modes before. This also demonstrates the importance of ultra-high energy resolution in EELS, (a)

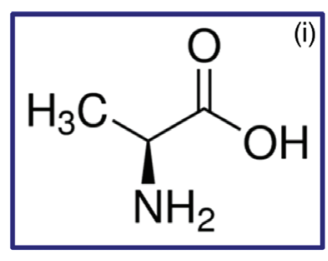

(b)

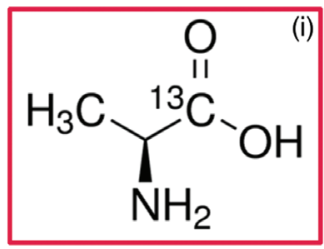

(c)

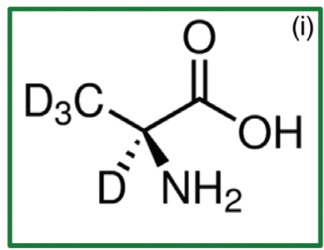

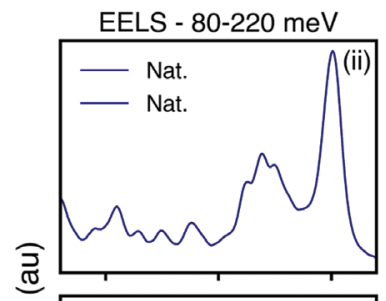

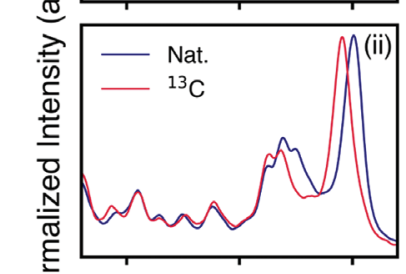

을

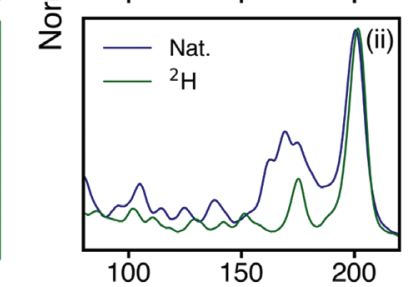

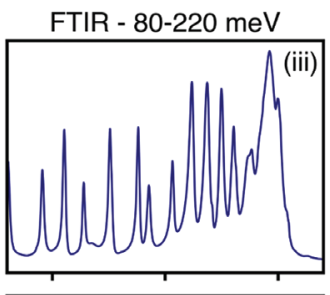
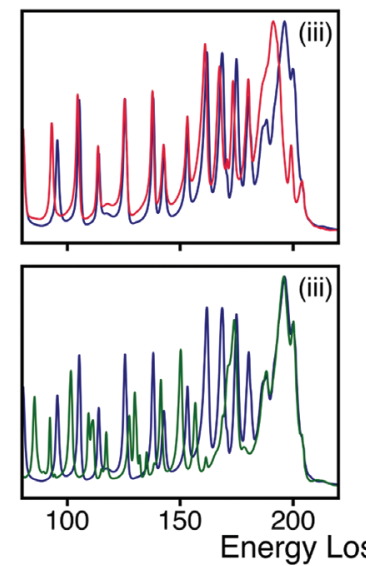
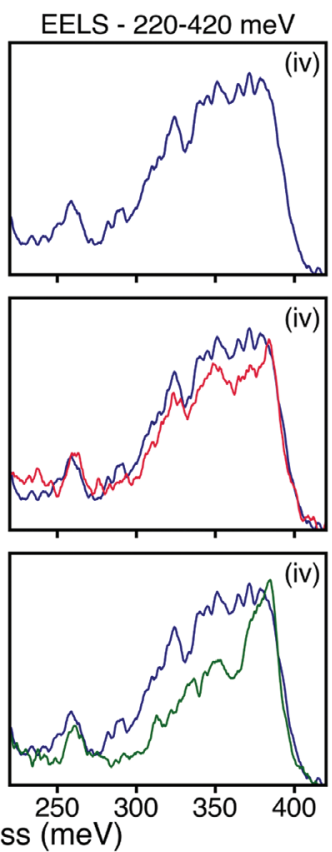
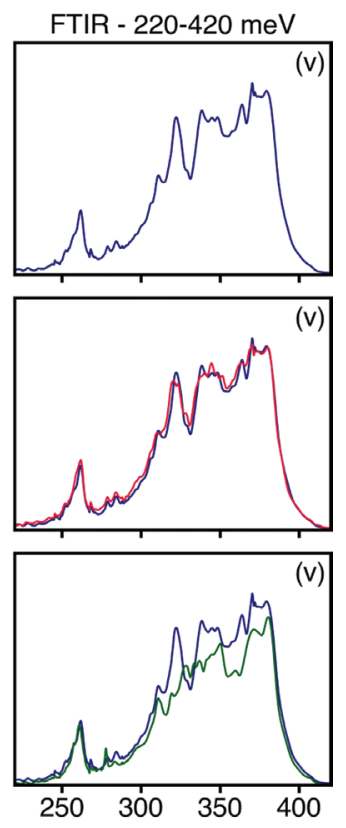

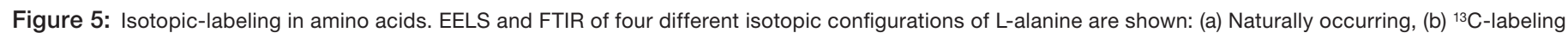

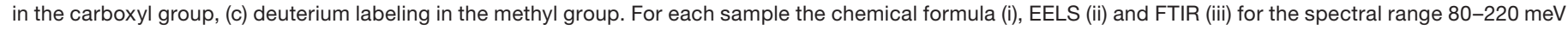
and EELS (iv) and FTIR (v) for the spectral range 220-420 meV are shown. Figure adapted from [12]. 
which is capable of resolving this vibrational fine structure. The isotopic resolution can be seen by comparing the natural L-alanine spectrum with the ${ }^{13} \mathrm{C}$-labeled L-alanine shown in the second row. In the 80-220 meV regime, a clear shift of the dominant peak at $\sim 200 \mathrm{meV}$ is present, as observed both in the FTIR and the EELS. This corresponds to the $\mathrm{C}-\mathrm{O}$ stretch mode in the carboxylate group [31], which is where the isotopic-label is in the molecule. The peak shifts by a factor of 1.026 (2.6\%), which matches closely to the change in the reduced mass of a $\mathrm{C}-\mathrm{O}$ pair with a ${ }^{13} \mathrm{C}$ instead of a ${ }^{12} \mathrm{C}\left(\sqrt{\mu^{13} \mathrm{CO} / \mu^{12} \mathrm{CO}}=1.022\right)$. Moreover, the measurement precision of the peak frequency is better than $0.2 \mathrm{meV}$, which both demonstrates the clear identification of isotopic labels and demonstrates that isotope-resolved spectroscopy could be pushed even further to heavier isotopes with smaller differences in the reduced mass while still being unambiguous.

While isotopic identification is simple for the case of ${ }^{13} \mathrm{C}$-labeling at the carboxyl site in L-alanine (and probably in many molecules where the $\mathrm{C}-\mathrm{O}$ stretch mode is a dominant vibrational feature), the results are often not so clearly understandable. For instance, the deuteration in the methyl group results in a much more complex change to the vibrational fine structure, as seen in Figure $5 \mathrm{c}$ (ii-v). In this molecule, 4 different atoms have changed their isotopic character (as opposed to one), and the difference in weight is more significant $\left(\sqrt{\mu C^{2} H / \mu C^{1} H}=1.363\right)$. Furthermore, the hydrogen atoms play a vital role in intermolecular bonding while the $\mathrm{C}-\mathrm{O}$ stretch mode does not; as a result, the $\mathrm{H}$ atoms contribute to many coupled modes through the response as opposed to being attributed to single definitive peaks. Thus, in the isotopically enriched molecule, the spectrum is significantly altered, to the extent where the vibrational fine structure is nearly unrecognizable. In fact, the only clear similarity between the naturally occurring alanine spectrum and the ${ }^{2} \mathrm{H}$-labeled spectrum is the ${ }^{12} \mathrm{C}-\mathrm{O}$ stretch mode.

While no significant changes were observed in the ${ }^{13} \mathrm{C}$-labeling for the 220-420 meV spectral range, we can observe significant changes due to the D labeling in both FTIR, Figure $5 \mathrm{c}$ (iv), and EELS, Figure $5 c(v)$. However, once again differences are observed, but there is no obvious shift or spectral feature that could be rigorously attributed to the deuteration, especially if a high degree of noise is present in the spectrum.

These results demonstrate that the vibrational response of a molecule is extremely sensitive to isotopic labeling, but interpretation of the response is not always straightforward. Therefore, while some atomic sites (carboxyl $\mathrm{C}$ atom) and vibrational modes (C-O stretch mode) allow for unambiguous identification of isotopic labels, other sites and other peaks do not. Future work will have to be rooted in theory with a clear understanding of the expected shifts due to the isotopic labels at deliberately chosen atomic sites.

\section{Spatially Resolved Identification of Isotope Labels}

Monochromated EELS now offers comparable spectral resolution to macroscopic optical techniques, and it is the nanoscale spatial resolution that drives the impact of the STEM/EELS approach. To demonstrate the spatial resolution of aloof vibrational EELS, we examine crystalline clusters of L-alanine with the different samples from Figure 5 dispersed on the same TEM grid and use the vibrational response to perform nanoscale identification of isotopic labels.

Figure 6 a shows a dark-field image of two clusters of alanine separated by several hundred $\mathrm{nm}$, one in its naturally occurring state $\left({ }^{12} \mathrm{C}\right.$, same as in Figure 5a) and one with the isotopic enrichment at the carboxyl carbon site $\left({ }^{13} \mathrm{C}\right.$, same as in Figure $\left.5 \mathrm{~b}\right)$. An EELS line scan was recorded, and each spectrum has the ZLP removed with a power law background subtraction and is plotted in Figure 6b. For each spectrum, the dominant $\mathrm{C}-\mathrm{O}$ stretch (at $200 \mathrm{meV}$ ) is fit with a Lorentzian function, and the peak energies are plotted in Figure $6 c$. Here, we observe that a redshift is induced by the increased mass in the isotopically labeled molecules as a function of probe position. Additionally, while the measured peak values in Figure $6 \mathrm{c}$ clearly stabilize at the ${ }^{12} \mathrm{C}-\mathrm{O}$ frequency (naturally occurring) to the ${ }^{13} \mathrm{C}-\mathrm{O}$ frequency (isotopically labeled) at each end of the line scan, the delocalization of aloof mode spectroscopy measures the $\mathrm{C}-\mathrm{O}$ frequency as a mixture of both, when the probe is between the two clusters. By calculating the strength of the interaction with each cluster as a function of separation of the two clusters, we estimate that aloof EELS can unambiguously determine the isotopic character of clusters separated by as little as $50 \mathrm{~nm}$.

\section{Conclusion}

Monochromated EELS presents a powerful new option in the analysis of biological materials. The ultra-high energy
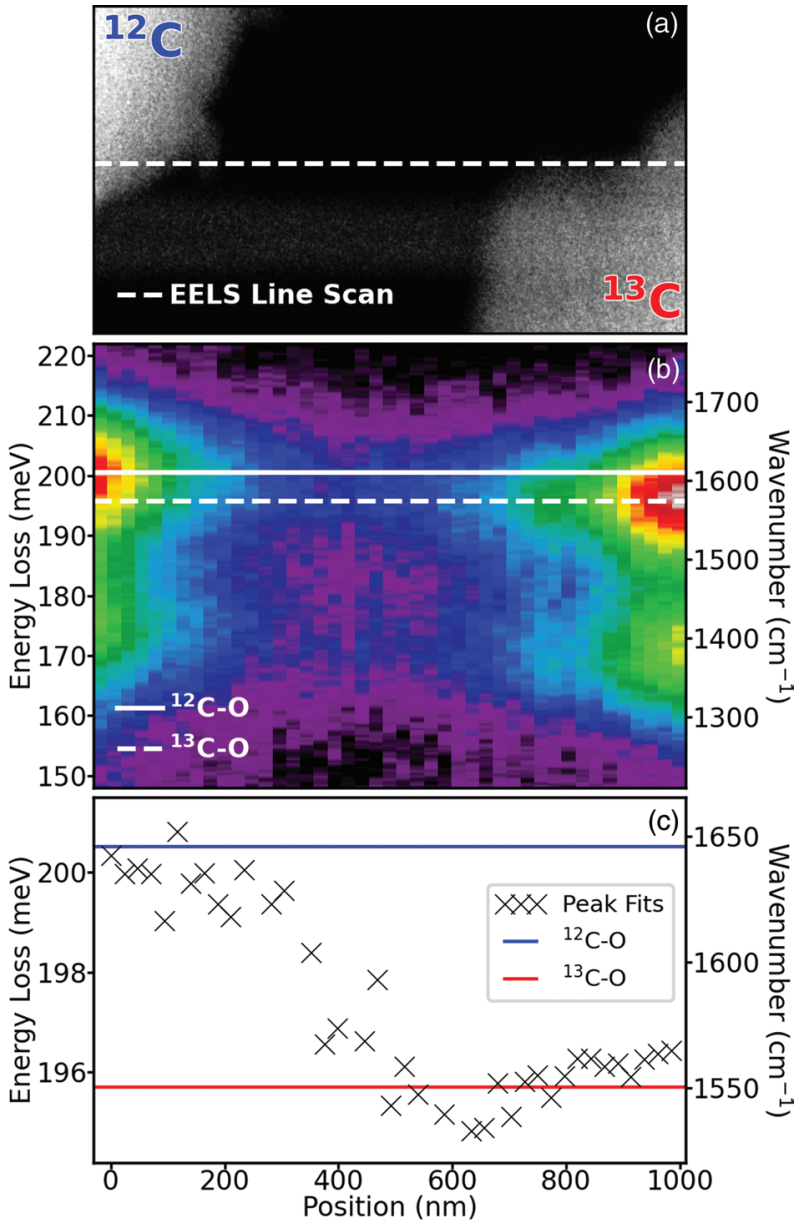

Figure 6: Spatially and isotopically resolved EELS. (a) Dark-field image of two alanine clusters: one with the natural occurring ${ }^{12} \mathrm{C}$ isotope in the carboxyl site (left), and one with the ${ }^{13} \mathrm{C}$ isotope (right). (b) The background-subtracted EELS from along the linescan, demonstrating the localization of the signal. (c) The dominant $200 \mathrm{meV}$ peak (corresponding to the C-O stretch mode in the carboxyl group) is fit for each spectrum demonstrating the real-space visualization of the isotopic-redshift. Figure adapted from [12]. 
resolution enables precise detection of the vibrational fine structure for straightforward identification of isotopic labels, and the aloof beam geometry enables beam-sensitive samples to be analyzed without incurring significant damage and sacrificing the nanometer spatial resolution. The examples shown here demonstrate the power of the technique, and they promise to lead to high spatial resolution analysis of whole-cell biological samples to enable real-space tracking of isotopically labeled molecules in the products of chemical reactions and metabolic pathways.

\section{Acknowledgements}

The authors would like to acknowledge Dr. Ilja Popovs of ORNL for his assistance with the FTIR data shown in Figure 5. Microscopy results shown here were supported by the Center for Nanophase Materials Sciences, which is a Department of Energy (DOE) Office of Science User Facility. Microscopy research was also performed, in part, using instrumentation within ORNL's Materials Characterization Core provided by UT-Battelle, LLC, under Contract No. DE-AC05- 00OR22725 with the DOE, and sponsored by the Laboratory Directed Research and Development Program of ORNL, managed by UT-Battelle, LLC, for the U.S. DOE. R.F.K. is supported in part by the Joint Center for Energy Storage Research (JCESR), an energy innovation hub funded by the U.S. DOE, Office of Science, Basic Energy Sciences.

\section{References}

[1] N Dellby et al., Microsc Microanal 26 (2020) in press.

[2] OL Krivanek et al., Nature 514 (2014) 209-12.

[3] MJ Lagos et al., Nature 543 (2017) 529-32.

[4] AA Govyadinov et al., Nat Commun 8 (2017) 1-10.
[5] Y Li et al., Sci Bull 65 (2020) 820-26.

[6] N Li et al., Nat Mater (2020) in press.

[7] SH Cho et al., Chem Mater 31 (2019) 2661-76.

[8] KC Smith et al., Phys Rev Lett 123 (2019) 177401.

[9] SH Cho et al., J Chem Phys 152 (2020) 1-17.

[10] P Rez et al., Nat Commun 7 (2016) 1-7.

[11] JR Jokisaari et al., Adv Mater 30 (2018) 1802702-1-6.

[12] JA Hachtel et al., Science 363 (2019) 525-28.

[13] SM Collins et al., Nano Lett 20 (2020) 1272-79.

[14] T Susi et al., Nat Commun 7 (2016) 13040-1-8.

[15] G Argentero et al., Ultramicrosc 151 (2015) 23-30.

[16] FS Hage et al., Phys Rev Lett 122 (2019) 016103-1-5.

[17] K Venkatraman et al., Nat Phys 15 (2019) 1237-41.

[18] FS Hage et al., Science 367 (2020) 1124-27.

[19] C Dwyer et al., Phys Rev Lett 117 (2016) 256101-1-5.

[20] PA Crozier, Ultramicroscopy 180 (Suppl C) (2017) 104-14.

[21] RF Egerton, Ultramicroscopy 180 (Suppl C) (2017) 115-24.

[22] J Atzrodt et al., Angewantde Chem Intl Ed 57 (2018) 1758-84.

[23] Z Wang et al., J Phys Chem A 108 (2004) 9054-63.

[24] HJ Bakker and JL Skinner, Chem Rev 110 (2010) 1498-1517.

[25] NM Schneider et al., J Phys Chem C 118 (2014) 22373-82.

[26] SM Ghodsi et al., Small Methods 3 (2019) 1900026.

[27] G Wu, Amino Acids 37 (2009) 1-17.

[28] OL Krivanek et al., Microscopy 62 (2013) 3-21.

[29] JA Hachtel et al., Sci Rep 8 (2018) 5637-46.

[30] PJ Gomes et al., J Phys Chem B 119 (2015) 5404-11.

[31] E Tajkhorshid et al., J Phys Chem B 102 (1998) 5899-5913.

\section{Fast, Easy and Affordable}

\section{From full-size to desktop, we have you covered.}

At COXEM, we believe that electron microscopy doesn't have to be complicated or expensive. Whether you are looking for a desktop or full-size SEM, COXEM electron microscopes are loaded with the features advanced users expect, at a price that entry-level users can afford. Call your local agent to arrange a demonstration, or visit our website for more information.

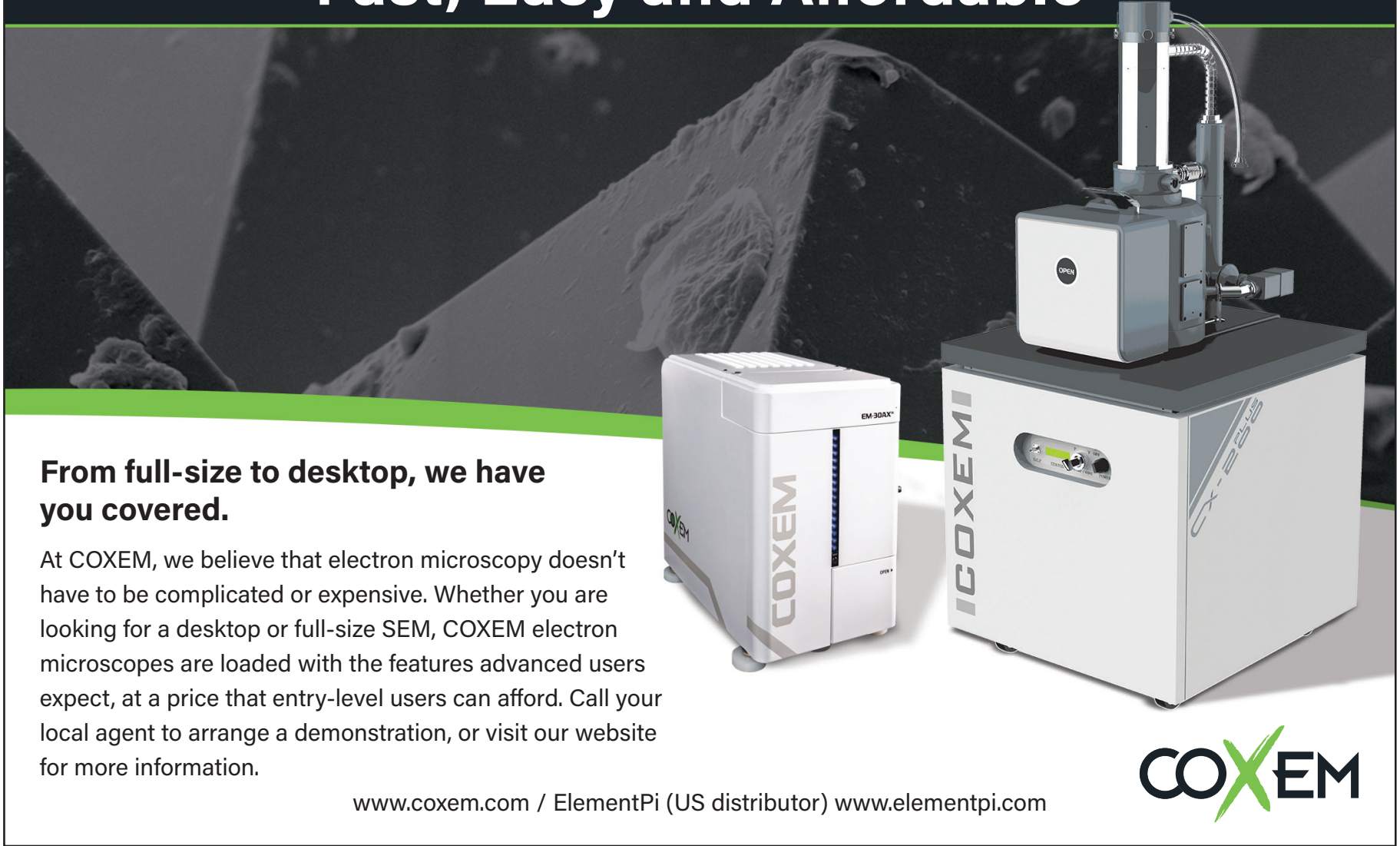

